\title{
Guidance to Employers on Integrating E-Cigarettes/Electronic Nicotine Delivery Systems Into Tobacco Worksite Policy
}

\author{
Laurie P. Whitsel, PhD, Neal Benowitz, MD, Aruni Bhatnagar, PhD, FAHA, Chris Bullen, MBChB, PhD, \\ Fred Goldstein, Lena Matthias-Gray, BS, Jessica Grossmeier, PhD, MPH, John Harris, MEd, \\ Fikry Isaac, MD, MPH, Ron Loeppke, MD, MPH, Marc Manley, MD, MPH, Karen Moseley, Ted Niemiec, MD, \\ Vince O'Brien, LaVaughn Palma-Davis, MA, Nico Pronk, PhD, Jim Pshock, Gregg M. Stave, MD, JD, MPH, \\ and Paul Terry, PhD
}

In recent years, new products have entered the marketplace that complicate decisions about tobacco control policies and prevention in the workplace. These products, called electronic cigarettes (e-cigarettes) or electronic nicotine delivery systems, most often deliver nicotine as an aerosol for inhalation, without combustion of tobacco. This new mode of nicotine delivery raises several questions about the safety of the product for the user, the effects of secondhand exposure, how the public use of these products should be handled within tobacco-free and smoke-free air policies, and how their use affects tobacco cessation programs, wellness incentives, and other initiatives to prevent and control tobacco use. In this article, we provide a background on e-cigarettes and then outline key policy recommendations for employers on how the use of these new devices should be managed within worksite tobacco prevention programs and control policies.

From the American Heart Association (Dr Whitsel), Washington, DC; University of California (Dr Benowitz), San Francisco; The University of Louisville (Dr Bhatnagar), Louisville, Ky; University of Auckland (Dr Bullen), Auckland, New Zealand; Population Health Alliance (Mr Goldstein), Washington, DC; University of Michigan (Ms Matthias-Gray and Ms PalmaDavis), Ann Arbor; Health Enhancement Research Organization (Dr Grossmeier), Edina, Minn; Performance pH (Mr Harris), Holland, Ohio; Johnson \& Johnson (Dr Isaac); US Preventive Medicine/American College of Occupational and Environmental Medicine (Dr Loeppke), Elk Grove Village, Ill; American College of Preventive Medicine (Dr Manley), Washington, DC; Health Enhancement Research Organization, Population Health Alliance (Ms Moseley), Edina, Minn; ArcelorMittal/American College of Occupational and Environmental Medicine (Dr Niemiec), Elk Grove Village, Ill; Interactive Health (Mr O'Brien); HealthPartners/ Harvard University (Dr Pronk); Bravo Wellness (Mr Pshock), Cleveland, Ohio; Prevention Partners/American College of Occupational and Environmental Medicine/Duke University (Dr Stave), Durham, NC; and StayWell Health Management (Dr Terry), Saint Paul, Minn.

The authors declare no conflicts of interest.

Address correspondence to: Laurie P. Whitsel, PhD,

Policy Research, American Heart Association,

1150 Connecticut Avenue, Suite 300, Washington, DC 20036.

Copyright (C) 2015 by American College of Occupational and Environmental Medicine

DOI: 10.1097/JOM.0000000000000420

\section{DESCRIPTION OF E-CIGARETTES}

Electronic cigarettes (e-cigarettes) are a general category of products that most often use battery power to heat a solution of tobacco-derived nicotine in propylene glycol and/or glycerol that is aerosolized for inhalation. ${ }^{1}$ Some e-cigarettes or e-cigarette liquids, however, contain only the carrier but no nicotine. Collectively, these devices are referred to as electronic nicotine delivery systems, a category that also includes devices referred to as personal vaporizers, vape pens, and e-hookah. Although there is significant difference in design features of these devices, their key components include a battery (often rechargeable), a heating element (atomizer), and the reservoir or cartridge for storing the liquid for aerosolization. Some of these devices are designed to appear similar to cigarettes (so-called cigalikes), although newer models are much larger and have a unique tank-like device (tankomizers) that can hold several milliliters of eliquid. ${ }^{2}$ Newer e-cigarettes also have programmable features to control heating temperature, which influences the extent of nicotine delivery. ${ }^{1}$ Some e-cigarettes are dispensable and others have replaceable cartridges. Although many cigalikes are fully closed and are disposable, the tankomizers and other electronic nicotine delivery systems come with cartridges or refillable tanks containing variable concentrations of nicotine in propylene glycol and/or glycerol (vegetable glycerin) and a wide range of optional flavoring agents including tobacco and menthol and fruit and candy. ${ }^{3}$ When users puff on the e-cigarette or, in some models, activate a switch, the liquid is heated and the resulting aerosol is available to be inhaled.

E-cigarettes are not currently regulated by the US Food and Drug Administration (FDA). In 2014, the FDA initiated a process that could lead to regulation of e-cigarettes under authority granted by the Family Smoking Prevention and Tobacco Control Act (FSPTCA). ${ }^{4}$ Although the FDA has approved several nicotine replacement products for tobacco cessation, e-cigarettes have not been approved by the FDA for this purpose. Moreover, even though many smokers report that using e-cigarettes assists in quitting smoking, 5,6 and two small clinical trials found that e-cigarettes promote long-term smoking cessation, ${ }^{6}$ there is not sufficient scientific evidence to suggest that they are effective and their relative efficacy in comparison with other FDAapproved nicotine replacement therapy needs further elucidation.

\section{E-CIGARETTES IN THE MARKETPLACE}

The first e-cigarette design was patented in $1965,{ }^{8}$ and the first product was introduced to the US marketplace in 2003 from China. ${ }^{1}$ Since then, the design of the devices has undergone considerable evolution and currently the marketplace has been flooded with a plethora of brands selling devices with many different design features. A 2014 survey of Web sites identified at least 466 brands available in 7764 different flavors, with two types of propellants, offering an average of four to five nicotine strengths. ${ }^{2}$ These products are sold on-line ${ }^{2}$ and in retail stores. ${ }^{9}$ In the United States, they are available in more than $30 \%$ of retail stores, most often in those specializing in tobacco products, but increasingly in convenience stores and other outlets. ${ }^{9}$ An audit of a nationally representative sample of US retailers found that e-cigarettes were more likely to be sold in neighborhoods with higher median household income and a lower percentage of minority residents. ${ }^{9}$ The sales of e-cigarettes in the United States have increased steadily since these products were first introduced in the market, and are predicted to reach at least $\$ 10$ billion by 2017 on the basis of current market trends, as well as the fact that advertising expenditures across all media outlets have surpassed $\$ 82$ million in 2013 . $^{10}$

\section{CONSTITUENTS, TOXICOLOGY, AND HEALTH EFFECTS OF E-CIGARETTES}

The major difference between ecigarettes and conventional cigarettes is that e-cigarettes do not generate a nicotine aerosol by combustion. Smoking conventional 
cigarettes involves the combustion of tobacco, which generates more than 5000 distinct chemicals dispersed in both the particulate and gas phase of the smoke. ${ }^{11}$ These include reactive and toxic carbonyls such as acrolein, acetaldehyde, butyraldehyde, formaldehyde, propionaldehyde, and butadiene, metals such as cadmium, lead, arsenic, and nickel, as well as carbon disulfide, hydrogen cyanide, benzene, nitrosamines, and polycyclic aromatic hydrocarbons (PAHs). Cigarette smoke also contains high levels of carbon monoxide (CO), which increases the levels of carboxyhemoglobin and reduces oxygen delivery from red blood cells in smokers. The particulate phase of mainstream smoke contains more than $5 \times 10^{25}$ particles per cubic $\mathrm{cm}^{12}$ that range in particle size from 0.1 to $1 \mu \mathrm{m}$. Recurrent exposure to this complex mixture of chemicals has been shown to result in the development of cancer, as well as cardiovascular and respiratory diseases, including an increased risk and severity of respiratory tract infections. ${ }^{13}$

Although there is little direct evidence linking individual chemicals to specific toxicological effects of tobacco smoke, exposure to tobacco smoke constituents such as carbonyls, benzene, butadiene, metals, tobacco-specific nitrosamines, and PAHs has been associated with various adverse health effects. ${ }^{14}$ Biomarkers of exposure to tobaccospecific nitrosamine and PAHs have been independently associated with the development of lung cancer in smokers. ${ }^{15}$

Results from animal studies suggest that exposure to aldehydes such as acrolein results in endothelial dysfunction, ${ }^{16}$ dyslipidemia, ${ }^{17}$ increased thrombosis, ${ }^{18}$ and increased formation of atherosclerotic lesions. ${ }^{19}$ Recent hazard index approaches developed from weighted exposure to individual mainstream smoke constituents suggest that much of the derived theoretical noncancer index is dominated by the reactive aldehyde acrolein whereas the theoretical cancer index is dominated by genotoxic carcinogens of the mainstream vapor phase such as 1,3-butadiene, isoprene, formaldehyde, acetaldehyde, and acrylonitrile. ${ }^{20}$

The levels of most of the chemicals with significant cancer and noncancer risk are much lower in e-cigarette liquid than in conventional cigarettes. ${ }^{21}$ Nevertheless, the levels of these chemicals generated in e-cigarette aerosol are variable and depend upon the specific device, the battery voltage, and puff duration because they affect the temperature at which the liquid will be heated. In addition to nicotine, e-cigarettes contain glycerol and propylene glycol also called propane-1,2-diol. Propylene glycol, a key component of e-cigarette liquids because of its ability to create a fine aerosol, when heated, is generally considered nontoxic ${ }^{22}$ and has been approved by the FDA as a solubilizing agent for different types of medications. ${ }^{23}$ It is also used to generate theater fog and in the aviation industry. Prolonged exposure to propylene glycol can cause eye and respiratory irritation. Therefore, frequent exposure to propylene glycol could be of concern, especially when the exposure involves susceptible individuals, such as those with asthma or chronic obstructive lung disease. In addition to propylene glycol and glycerol, ethylene glycol has also been detected in some e-cigarette aerosol. ${ }^{24}$ Ethylene glycol is used in antifreeze and other industrial formulations and it is a strong irritant with moderate toxicity that affects the central nervous system and cardiovascular tissues. Hence, its use as a humectant in conventional tobacco products is currently prohibited. ${ }^{24}$ Ingestion of a high amount of ethylene glycol can be fatal. ${ }^{25}$

Because nicotine in most e-cigarette liquids is derived from tobacco, it contains trace levels of tobacco alkaloids such as nornicotine, anabasine, and anatabine as well as tobacco-specific nitrosamines including $\mathrm{N}$-nitrosonornicotine and 4-(methylnitrosamine)-1-(3-pyridyl)1-butanone $(\mathrm{NNK}){ }^{26}$ In most samples of e-cigarette liquids, the levels of minor tobacco alkaloids such as nornicotine, anatabine, and anabasine are between $1 \%$ and $2 \%$ of nicotine. These alkaloids have actions similar to nicotine but are generally less toxic and less potent, and their presence in trace levels is not currently believed to significantly impact e-cigarette toxicity. ${ }^{27}$ The main e-cigarette constituents of concern, however, are volatile organic compounds and particulate matter. In conventional cigarettes, these constituents are generated by combustion, and even though heating and vaporization of e-cigarette liquids do not involve combustion, high levels of organic compounds such as formaldehyde have been detected in some e-cigarette aerosols with higher levels seen with higher vaporization temperatures. ${ }^{28}$ Nonaerosolized samples of e-cigarette liquids contain only trace levels of aldehydes; however, 10- to 20-fold higher concentrations of aldehydes are generated upon heating. ${ }^{24}$ Aldehyde generation is further accelerated by increased air flow, and therefore the release of aldehydes is significantly increased as the liquid level in the cartridge is decreased and replaced by air. Therefore, in some conditions (eg, after 10 to 100 puffs), the levels of aldehydes generated in e-cigarettes are comparable to or even higher than those in conventional cigarettes. $^{24}$ Similarly, aerosol generated by e-cigarettes contains particulates at a number, concentration, and size similar to those of conventional cigarettes. ${ }^{29,30}$ Extensive studies have shown that exposure to ambient air particles of the size distribution similar to those in e-cigarettes is associated with significant cardiopulmonary toxicity and mortality. ${ }^{31}$ Nevertheless, unlike ambient air particles, e-cigarette particles do not contain carbon and are generated as an aerosol from supersaturated propane-1,2-diol vapor. There is no evidence available to indicate whether their toxicity is similar to that of ambient air particles or particles generated in tobacco smoke.

There have been few direct evaluations of the health effects and toxicity of e-cigarettes on humans or animals. Acute exposure in individuals using e-cigarettes has been found to increase dynamic air resistance and to significantly decrease exhaled nitric oxide, ${ }^{32}$ indicating that e-cigarette constituents may be pulmonary irritants. Nevertheless, unlike conventional cigarettes, using e-cigarettes has not been associated with a decrease in forced expiratory volume (FEV) or an increase in inflammatory responses. ${ }^{33}$ The use of e-cigarettes has been associated with an acute increase in heart rate and blood pressure. Nevertheless, the changes in diastolic function and increased coronary vascular resistance seen with smoking conventional cigarettes have not been observed. ${ }^{34}$ In randomized controlled studies, no serious adverse health effects have been reported in individuals using e-cigarettes for the 6-month duration of the research protocol. ${ }^{34}$ Studies with longer follow-up have not been performed. Asthmatic smokers switching from conventional cigarettes to e-cigarettes have reported improvements in $\mathrm{FEV}_{1}$ and scores on an asthma control questionnaire, with most of the improvement likely related to quitting smoking or a decrease in the number of cigarettes smoked per day. ${ }^{35}$ Several anecdotal incidences, such as an increase in atrial fibrillation, ${ }^{36}$ have also been reported. Some flavorings in e-cigarettes contain diacetyl, a compound that is known to cause bronchiolitis obliterans, a condition that may result in respiratory failure and require lung transplantation. ${ }^{37}$ Further studies will be required to establish the biological plausibility of these effects and to ascertain their prevalence in e-cigarette users.

In summary, even though the specific toxicity of e-cigarette constituents remains unclear, because their emissions contain high levels of aldehydes and particulate matter (PM) with an aerodynamic diameter of less than $2.5 \mu \mathrm{m}$, there is concern that the frequent use of e-cigarettes could have adverse health effects. Further research and monitoring are required to assess both the short- and long-term toxicity of direct and secondhand exposure to e-cigarettes.

\section{Adult Awareness and Use of E-Cigarettes}

Awareness and use of e-cigarettes have increased significantly since researchers started monitoring US trends about 5 years ago, particularly among young adults. A 2011 
US consumer survey found that $6.2 \%$ of American adults have tried e-cigarettes at least once, a significant increase over 2010 levels. ${ }^{38} \mathrm{~A}$ review of studies indicates that the use of e-cigarettes has increased from less than $1 \%$ in 2009 to $6 \%$ in 2011 , with people trying them most often being current or former smokers. ${ }^{38-40}$ A recent study indicated that ever use of e-cigarettes doubled by 18 to 34 -year-olds from 2011 to $2012 .^{41}$

Awareness of e-cigarettes varies within the demographic profile of users. Men aged 45 to 54 years, with some college education, earning more than $\$ 60,000$ annually tend to be most aware of e-cigarettes. ${ }^{38}$ Ever-users tend to be female and aged 18 to 24 years, have a high school level of education, and earn $\$ 25,000$ to $\$ 39,999$ annually. ${ }^{38}$ These groups are more alike in their racial/ethnic background (more frequently white, non-Hispanic), have Midwestern residential status, and are current cigarette smokers. $^{38}$

\section{Youth Awareness and Use of E-cigarettes}

From an employer's perspective, young people represent the workforce of the future. The current use of e-cigarettes among young people could increase the likelihood of smoking in adolescence and adulthood. ${ }^{42}$ Because experimentation with e-cigarettes might lead to later or concurrent use of conventional cigarettes, it is important to prevent young people from obtaining and using e-cigarettes.

Data obtained from the US National Youth Tobacco Survey ${ }^{42}$ show an increase in ever use (at least one time in the last 30 days) of e-cigarettes among never-smoking young people from 79,000 in 2011 to 263,000 in 2013, a 3-fold increase. More than a quarter of a million youth who had never smoked a conventional cigarette used e-cigarettes in 2013. The researchers noted that e-cigarette use was associated with increased intentions to smoke cigarettes. Compared with those who had never used e-cigarettes, young individuals who had never smoked conventional cigarettes but who used e-cigarettes were almost twice as likely to have the intention to smoke conventional cigarettes. Almost $45 \%$ of nonsmoking youth who had ever used e-cigarettes indicated that they intend to smoke conventional cigarettes within the next year, compared with about $20 \%$ of those who had never used e-cigarettes, indicating that using e-cigarettes is correlated with the intention to smoke conventional cigarettes. The need for stringent regulations to prevent e-cigarette marketing and promotion to youths has been emphasized by the World Health Organization ${ }^{43}$ and the American Heart Association, ${ }^{44}$ especially given that the products come in attractive flavors and are comparatively cheaper than cigarette smoking. Both these factors make e-cigarettes particularly attractive to youth.

In addition to the impact of youth using e-cigarettes themselves, the use of e-cigarettes by adults poses additional health hazards to children. Through December 31, 2014, the American Association of Poison Control Centers had received calls reporting 2,724 e-cigarette device and liquid nicotine exposures. ${ }^{45}$ Slightly more than half of these reported exposures have occurred in children younger than 6 years and some children and toddlers have required emergency department visits with nausea and vomiting being the most significant symptoms. A toddler in upstate New York died from ingesting nicotine liquid, ${ }^{46}$ renewing calls to require child-proof packaging and adequate warning labels to prevent these tragedies.

\section{WORKPLACE EXPOSURE}

There is extensive, rigorous, and convincing evidence that secondhand exposure to cigarette smoke increases the risk of adverse health effects on others. ${ }^{13}$ Exposure to secondhand smoke has been linked to 600,000 premature deaths every year around the world, mostly from cardiovascular disease, asthma, lung cancer, and respiratory tract infections. ${ }^{47}$ Implementation of smoking bans in public places has led to an improvement in air quality and a significant decrease in the rate of acute cardiovascular events. ${ }^{14}$

Whether secondhand exposure to e-cigarettes has similar consequences remains unclear as only limited research has been completed on the secondhand effects of e-cigarettes. Unlike conventional cigarettes, e-cigarettes do not produce a side stream vapor or smoke that is generated from product smoldering. The emissions from e-cigarettes derive only from exhalation and contain e-cigarette constituents transformed after interaction with the human lung as well as other breath constituents of the user.

The limited evaluation of exhaled aerosol from e-cigarette users has shown great variability due to different styles of use, the e-cigarette types and products used, the characteristics of the indoor environment (closed or open) and the measurement methods used. Nevertheless, they show significant dispersion of propylene glycol and nicotine into the environment. In one study, measurements of the environment in which e-cigarette aerosol was exhaled showed significant levels of nicotine, propylene glycol in the gas phase, as well as high concentrations of $\mathrm{PM}_{2.5}$ (mean $\left.197 \mu \mathrm{g} / \mathrm{m}^{3}\right){ }^{48}$

Some studies have found that the exhaled e-cigarette vapor also contains volatile organic carbons as well as PAHs and aluminum. No increase in the levels of $\mathrm{CO}$, or potentially carcinogenic elements such as cadmium, arsenic, and thallium, has been detected. ${ }^{49}$ In one study, the use of nicotinecontaining e-cigarettes in a ventilated room for 2 hours did not result in an increase in formaldehyde, benzene, or acrolein levels. ${ }^{48}$

The presence of nicotine in exhaled ecigarette vapor could potentially expose individuals in the vicinity to significant levels of nicotine. In a human exposure study in which 15 individuals participated in a passive e-cigarette use session, exposure to ecigarette vapor for 1 hour increased serum cotinine levels similarly to those observed with secondhand exposure to combustible tobacco smoke, ${ }^{33}$ suggesting that significant secondhand exposure to e-cigarette emissions could lead to significant passive absorption of nicotine. Although the effects of exposure to secondhand nicotine have not been studied directly, these findings also suggest that there is the potential for significant involuntary exposure in a bystander as a result of being near someone who is using an e-cigarette.

Passive exposure to particulates generated in exhaled e-cigarette aerosols could also potentially have adverse health consequences. E-cigarette aerosols have been found to contain fine and ultrafine particles. These particles show a bimodal distribution with peaks at 30 and $100 \mathrm{~nm}$. In comparison, the particle size distribution of the conventional cigarette shows a single mode with a maximum at $100 \mathrm{~nm} .{ }^{50}$ Although in some studies the number concentration of particles generated from e-cigarettes has been found to be similar to that of conventional cigarettes, it is important to note that e-cigarette-derived particles do not contain carbonaceous material. They are also likely to be vastly different in their chemical properties from the particles emitted by combustible cigarettes or those present in the ambient air, which have been linked to a variety of untoward health effects. ${ }^{31}$ Environmental e-cigarette particles are exhaled after inhalation, and therefore it is likely that their size distribution and other properties are altered in the human lung, which leads to the exhalation of smaller particles with altered toxicological properties. ${ }^{50}$ Further studies are required to address these issues.

\section{APPROACHES USED BY DIFFERENT COUNTRIES IN CREATING E-CIGARETTE POLICIES}

E-cigarettes have fallen between existing regulatory gaps. Because of their novelty and the lack of conclusive evidence on their health effects, safety, and cessation efficacy, it has been unclear whether e-cigarettes should be regulated as tobacco products, therapeutic goods, medical devices, or consumer 
"lifestyle" products. As a result, the development of an effective regulatory policy is likely to be a complex undertaking. Ultimately, the chosen regulatory route will depend on many factors, including the flexibility of the current regulatory framework, whether the products have significant health effects and toxicity, and whether therapeutic claims are successfully made by the manufacturers (eg, the use of e-cigarettes as a cessation aid).

Currently, the regulation of ecigarettes varies widely around the world, from an absence of any regulation (eg, in Turkey) to absolute bans on use, sales, and marketing (eg, in Singapore and Canada). Many countries make a distinction between nicotine and non-nicotine products, whereby refills or cartridges without nicotine are permitted (as long as they do not make therapeutic claims) but those with nicotine require a license. Most countries that regulate e-cigarettes allow the importation of devices and liquids for personal use but ban importation for sale. Few countries have yet determined whether or not e-cigarettes should be subject to regulatory controls when used in smoke-free areas, such as workplaces.

In the United Kingdom, e-cigarettes are regulated as a medicine and a product that is currently undergoing review by the medicines regulator. Other restrictions, including the age of individuals permitted to purchase, and use where smoking is currently banned, are under consideration. ${ }^{51}$ In the European Union, e-cigarettes have been categorized as tobacco products. E-cigarettes were included in the recent European Union Tobacco Products Directive that proposed banning devices, refills over 20-mg nicotine strength, advertising, and international sales over the Internet. $^{52}$

In August 2014, the World Health Organization recommended that governments ban e-cigarette use indoors, prohibit sales to people younger than 18 years, ban the use of vending machine sales, and restrict manufacturers from claiming e-cigarettes aid smoking cessation until they provide evidence to support such claims. ${ }^{43}$

The recommendations in this policy paper apply to the United States, where ecigarettes are not currently regulated by the FDA. After an unsuccessful attempt to regulate e-cigarettes as drug delivery devices, the FDA now intends to regulate e-cigarettes as "tobacco products," not as drugs or delivery devices unless marketed for therapeutic purposes. This was the result of a clarification of the FDA's authority under the 2009 FSPTCA. ${ }^{53}$ Among the regulations proposed are disclosure of ingredients, proof of safety, and regulation of the design features of the devices.

The FSPTCA does not propose overriding the ability of states or local authorities to make laws and policies related to e-cigarettes. In the absence of federal regulations, more than half of US states and municipalities have proceeded to enact their own e-cigarette regulations. ${ }^{53}$ Most commonly, these laws prohibit sales to minors, while some laws prohibit e-cigarette use in areas where smoking is banned.

\section{HOW US EMPLOYERS SHOULD ADDRESS E-CIGARETTES}

\section{Policy Recommendations}

The following sections outline key recommendations for employers as they develop policies regulating the use of ecigarettes in the workplace (Table 1). These recommendations do not constitute legal advice. Employers should consult with their attorneys to ensure compliance with applicable local, state, and federal laws. Employers should review applicable state and local laws regulating e-cigarette use that might already exist in their cities or states. It is also recommended that employers at least consider e-cigarettes that contain nicotine to be tobacco products and develop policies consistent with this perspective. ${ }^{44}$

\section{Tobacco-Free Policies}

\section{Recommendation}

Employers should include e-cigarettes in their tobacco-free policies and should ban e-cigarette use in their smoke-free work areas.

\section{Explanation}

Tobacco-free and clean indoor air policies continue to become more prevalent in US workplaces. More than 24 states have banned smoking in the workplace, but a majority of these have not included e-cigarettes in their laws. ${ }^{54}$ This is particularly significant, as e-cigarettes are becoming more popular and distributors continue to market them as a way to circumvent smoke-free laws, claiming that they contain "no tobacco smoke, only vapor.",3,55

At a distance, e-cigarette use can be difficult to distinguish from conventional cigarette use and therefore can create confusion in smoke-free areas. ${ }^{56}$ The inability of employers to distinguish between conventional and e-cigarettes may make it difficult to monitor employee cigarette use and to implement and enforce tobacco-free policies. ${ }^{44,57}$ A lack of enforcement of e-cigarettes in tobacco-free areas may turn back decades of work to create social norms around clean indoor air in public places. ${ }^{44,58-60}$ Decreasing the visibility of cigarettes in the work environment and in the media has been a major factor in denormalizing smoking behavior. Therefore, reintroduction of smoking-like activities could erode these important public health gains.
For those organizations that continue to host designated cigarette smoking areas, we strongly recommend that they join the growing number of organizations with entirely tobacco-free buildings as well as those businesses who now support tobacco-free grounds. Nevertheless, if an organization is not yet ready to move to an entirely tobaccofree policy, we recommend that the organization create a separate designated e-cigarette area from the tobacco smoking area, given the significance of the evidence concerning the dangers of secondhand tobacco smoke detailed earlier. Also, it might be difficult for e-cigarette users to quit as recurrent exposure to tobacco smoke may tempt them to start smoking again.

\section{E-Cigarettes and Tobacco Cessation Benefits in Employer-Based Health Insurance}

\section{Recommendation}

Employers should offer comprehensive tobacco cessation services within their employee health care plans and wellness programs and e-cigarette users should be eligible for these programs.

\section{Explanation}

To address e-cigarette use, employers should examine their companies' tobacco cessation insurance benefits. Currently, federal law requires companies with nongrandfathered health plans to provide coverage for tobacco cessation as a preventive service. ${ }^{61}$ Nevertheless, policies to date are silent on whether e-cigarette users should be included under these benefits. The US Preventive Services Task Force has concluded that tobacco cessation services are effective, and that these services should include both counseling and FDA-approved pharmacotherapy. ${ }^{62}$ The Community Preventive Services Task Force provides several recommendations specific to the workplace setting. Smoke-free policies to reduce secondhand smoke exposure and tobacco use are recommended on the basis of strong evidence of effectiveness. In addition, the Community Preventive Services Task Force recommends worksite-based incentives and competitions when combined with additional interventions to support individual cessation efforts based on strong evidence of effectiveness in reducing tobacco use among workers. ${ }^{63}$ The US Department of Labor recently provided guidance to employers to help define adequate coverage as follows:

The Departments will consider a group health plan or health insurance issuer to be in compliance with the requirement to cover tobacco use counseling and interventions, if, for example, the plan or issuer covers without cost-sharing: 
TABLE 1. Summary Recommendations for US Employers to Address E-Cigarettes ${ }^{\mathrm{a}}$

\section{Recommendation}

Explanation/Rationale

Designate e-cigarettes that contain nicotine to be tobacco products

Review applicable state and local laws regulating e-cigarette use that might already exist

Employers should include e-cigarettes in their tobacco-free policies and ban e-cigarette use in their smoke-free work areas

Employers should offer comprehensive tobacco cessation services within their employee health care plans and wellness programs, and e-cigarette users should be eligible for these programs.

Employers should screen for both tobacco and e-cigarette use to tailor their wellness programs and worksite policies optimally.

Employers should target tobacco use instead of nicotine use if they elect to administer health contingent incentives. An incentive policy consistent with Affordable Care Act (ACA) regulation cannot require success at cessation under a health contingent incentive design but may offer employees who use tobacco "reasonable alternatives" that most commonly include completion of a cessation program.
This would be consistent with the way e-cigarettes have been treated under federal law. ${ }^{77}$

Company policy must be consistent with federal, state, and local laws. Although federal law is consistent, state and local laws vary.

The inability to distinguish between conventional and e-cigarettes makes it difficult to monitor and enforce compliance if e-cigarettes are not treated the same way as conventional cigarettes in smoke-free areas.

Failing to eliminate the use of e-cigarettes in smoke-free areas could turn back decades of work to create social norms around clean indoor air in public places.

Decreasing the visibility of cigarettes in the work environment and in the media has been a major factor in denormalizing smoking behavior; reintroduction of smoking-like activities could reverse important public health gains.

We recommend that organizations be entirely tobacco free in their buildings, but for those organizations that continue to host designated smoking areas, they should create a separate designated e-cigarette use area.

Many e-cigarette users also use other forms of tobacco and thus can be helped by cessation support.

Nicotine addiction is not a recognized disability under federal or most state laws, so employers are not obligated to allow e-cigarettes in the workplace to accommodate employees who are trying to quit smoking.

The US Preventive Services Task Force has concluded that tobacco cessation services are effective and should include both counseling and FDA-approved pharmacotherapy.

The Community Preventive Services Task Force recommends incentives and competitions along with other interventions to support cessation efforts based on strong evidence of effectiveness.

The US Department of Labor recently provided guidance to employers to help define adequate tobacco cessation coverage.

Screening can be performed by personal attestation or with biological testing of nicotine metabolites in the saliva, blood, or urine.

Biological testing has several limitations.

Employers who choose biological testing should have protocols in place to determine how to interpret positive findings, respond to false positives, and administer an appeals process.

If employers have concerns about the veracity of personal attestations, they may require a statement from the personal physician of the employee and/or a written voucher with legal sanctions for inaccurate reporting.

The ACA wellness regulations categorize "tobacco use" as a "health-contingent outcomes-based" category with which employers may associate financial rewards or penalties, as long as they comply with all of the requirements associated with such programs.

In addition to other requirements, the regulations limit the amount of the reward or penalty for tobacco use to $50 \%$ of the total premium for an employee's category (single, family, etc).

Although verification of tobacco use in the form of a blood or urine test for nicotine/cotinine is permitted, the fact that a positive result indicates only the presence of nicotine rather than tobacco, may pose a risk to employers who seek to tie the health-contingent incentive to the test. Determining tobacco use, electronic cigarette use, or use of a nicotine replacement therapy (NRT) will typically require an employee's personal attestation or affidavit or a note from his or her personal physician.

In a health-contingent incentive, a positive nicotine test would prevent an individual from earning an incentive (or avoiding a penalty) unless they meet the reasonable alternative standard and/or have provided a physician statement indicating that stopping the use of tobacco/nicotine or completing a cessation program is medically inadvisable or unreasonably difficult due to a medical condition or due to the use of an NRT. 
TABLE 1. (Continued)

\section{Recommendation}

Health care plans sold through the regional exchanges should include comprehensive tobacco cessation services that also address e-cigarettes in their coverage.

Joint labor-management actions should be taken to address e-cigarettes within tobacco control policies and programming at the workplace as part of the bargaining process.

Where employers extend their health promotion programs to families, they should raise awareness and include education around e-cigarettes and cessation therapy.

Employers should publicize their tobacco policies (including e-cigarettes) to demonstrate best-practice, comprehensive efforts that are a model in the community.

\section{Explanation/Rationale}

Regional exchanges may play a greater role as some employers shift their employees to the exchanges for health care coverage.

Employers should be deliberate about the tobacco cessation benefits they want to see offered in the exchanges for their employees and should decide which exchanges provide the best option for their employees' health and well-being.

Labor organizations are important, valuable, and culturally relevant partners in addressing e-cigarettes at the workplace.

Most unionized workers support their union in bargaining for restrictions on workplace smoking and for the availability of cessation programs.

There are many supportive actions labor organizations can take to help reduce worker exposure to nicotine and tobacco-related products.

It is especially important to reach children and adolescents where education may prevent initiation of a nicotine habit

Family members often drive more of the medical cost than employees.

Employers can help the community and the field gain a better understanding of best practices.

Employers can help inform further research around effective policy for e-cigarettes and other emerging tobacco products.

${ }^{a}$ This does not constitute legal advice. Employers should consult with their attorney(s) to comply with applicable local, state, and federal law.

1. Screening for tobacco use; and,

2. For those who use tobacco products, at least two tobacco cessation attempts per year. For this purpose, covering a cessation attempt includes coverage for:

- Four tobacco cessation counseling sessions of at least 10 minutes each (including telephone counseling, group counseling and individual counseling) without prior authorization; and

- All FDA-approved tobacco cessation medications (including both prescription and over-thecounter medications) for a 90 day treatment regimen when prescribed by a health care provider without prior authorization. ${ }^{64}$

In addition, it is important for employers to understand that tobacco cessation interventions are more likely to be successful when offered in a supportive work environment. Such an environment may include a policy that prohibits tobacco use on the property, incentives (financial and otherwise) to encourage cessation, and regular communications from company leadership about the benefits and importance of cessation.

Although the aforementioned federal guidelines do not address coverage of ecigarette users, we recommend that employers include them in their cessation program efforts. Many e-cigarette users also use other forms of tobacco, such as cigarettes. We recommend that any user of e-cigarettes, indulging in sole or dual use, has access to and coverage by the company's tobacco cessation program. The programs that support cessation among tobacco users are likely to benefit e-cigarette users as well.

As described in the introductory paragraphs of this article, both e-cigarette retailers and many e-cigarette users have claimed that e-cigarettes can play a role in smoking cessation. Absent definitive scientific evidence supporting such claims, we do not recommend the use of e-cigarettes as a part of smoking cessation programs. These recommendations may change if, and when, rigorous studies demonstrate the safety and efficacy of e-cigarettes in promoting cessation, and they are approved by the FDA as a cessation aid. Related to this, because e-cigarettes are not approved by the FDA as cessation aids, we do not recommend that e-cigarettes be offered as a covered benefit for tobacco users. Moreover, because nicotine addiction is not a recognized disability under federal or most state laws, employers are not obligated to allow e-cigarettes in the workplace to accommodate an employee who is trying to quit smoking. ${ }^{65}$

\section{Screening for E-Cigarettes}

\section{Recommendation}

Employers should screen for e-cigarette use in their health screenings and in their health insurance plan design and wellness programs to be able to tailor their wellness programs and worksite policies optimally.

\section{Explanation}

There are different ways to screen for nicotine and tobacco use, including personal attestation and biomarker testing. Most employers use personal attestation and some in- dicate that a falsified statement may be subject to disciplinary action. Alternatively, or additionally, biological testing can be considered. Biological testing for tobacco use typically involves the measurement of cotinine, the major metabolite of nicotine, usually in the urine, blood, or saliva. Employers who choose to measure cotinine should be aware that the test has several limitations and the results have to be interpreted with caution. The cutoff level for a positive test should be set high enough to avoid classifying people exposed only to environmental tobacco smoke as smokers. Tobacco product users can produce a negative cotinine test if they abstain from using the product for more than 4 days. ${ }^{66}$ It is also important to note that cotinine measurement in the blood, urine, or saliva cannot distinguish between a cigarette smoker, an e-cigarette user, or someone who is using other tobacco products or FDA-approved nicotine replacement therapy because all of these products contain nicotine, which upon metabolism generates cotinine. $^{44}$

Other biomarkers that have been considered for assessing tobacco use are anabasine and anatabine. Testing for anabasine can distinguish between the use of nicotine replacement therapy (NRT) from tobacco products. ${ }^{64}$ Measurable but low levels of anabasine and anatabine have been detected in a majority of e-cigarettes tested by the FDA, so anabasine/anatabine analysis might be able to distinguish between users of NRT and e-cigarettes. ${ }^{65}$ Nevertheless, whether e-cigarette use can be detected by anabasine/anatabine testing will depend on various factors, including the concentration of these minor alkaloids in the specific 
product and the time since last use. Tests are under development to better differentiate between e-cigarettes and cigarettes.

Clearly, a robust detection method would help distinguish between the uses of different products containing nicotine, but it is prudent to recognize the limitation of current assays. Employers who choose to pursue biological testing should have protocols in place to determine how to interpret positive findings, respond to false positives, and administer an appeals process. As mentioned previously, any testing must be in compliance with federal and state laws. South Carolina, for example, prohibits mandatory nicotine and tobacco testing by employers. ${ }^{67}$

A National Business Group on Health survey in 2012 indicated that $16 \%$ of members responding to the survey were using cotinine testing. ${ }^{68}$ With the provisions in the Affordable Care Act (ACA) that allow differential premiums based on health outcomes, the number of employers testing tobacco product use by their employees is likely to rise. Nevertheless, before testing, employers should consider the nature of the relationship they wish to have with their employees and the culture of their workplaces. Mandating biological testing for tobacco and nicotine use has the potential to result in a loss of trust between employers and workers. In addition, cost-effectiveness considerations factor into decision making concerning screening for tobacco use, given that large populations with a low percentage of tobacco users may find it undesirable to request invasive tests from the majority to validate the veracity of a minority. A simple attestation can be sufficient for qualifying the vast majority of nonsmokers for insurance policy differentials. If an organization remains concerned that a minority are inappropriately qualifying for insurance differentials, an attestation accompanied by a note from the employee's physician and/or a written voucher with legal sanctions for inaccurate reporting could be considered. Some employers have conducted outbound recorded phone calls with scripted messaging about the importance of truthful attestation as a way to reduce inaccurate employee self-reported nonsmoking.

\section{Addressing E-Cigarettes Within ACA Sanctioned Health Contingent, Outcomes-Based Incentive Programs}

\section{Recommendation}

Employers should target tobacco use instead of nicotine use if they elect to administer health contingent incentives in coordination with their wellness programs. An incentive policy consistent with ACA regulations cannot require success at cessation under a health contingent incentive design. Rather, it may offer employees who use tobacco "rea- sonable alternatives" the most common being the completion of a cessation program.

\section{Explanation}

The ACA wellness regulations ${ }^{69}$ categorize "tobacco use" as a "health-contingent" category with which employers may associate financial rewards or penalties (in the form of premium differentials or health benefit adjustments such as deductibles or copays), as long as they comply with all of the requirements associated with such incentive designs. Previous guidance has outlined how employers might consider designing their health contingent programs. ${ }^{70}$

In addition to requirements such as the incentive being part of a comprehensive wellness program that has a reasonable chance of improving the health of the individual, the final regulations limit the amount of the reward or penalty to $50 \%$ of the total employee-only premium (or $50 \%$ of the category in which the employee is enrolled if other family members are also part of the incentive design). The total of all rewards may not exceed the $50 \%$ threshold, and outside of tobacco use, the total of all categories may not exceed $30 \%$. Although verification of tobacco use in the form of a blood or urine test for nicotine/cotinine (or other metabolite of nicotine) is permitted, the fact that a positive result indicates only the presence of nicotine rather than tobacco may pose a risk to employers who seek to tie more than $30 \%$ of premium to the test. Determining true "tobacco use" will typically require an employee's personal attestation through a questionnaire or an affidavit, or a note from his or her personal physician. If the use is solely determined by the presence of nicotine in a laboratory test, employers are advised that it may be from NRT rather than tobacco use for purposes of determining the maximum reward or penalty. Employers are, however, permitted to require verification that the NRT is being used in conjunction with a qualified cessation program. ${ }^{69}$

The ACA wellness provisions related to the use of incentives specifically state that recommendations of an individual's personal physician must be accommodated with respect to "reasonable alternatives" related to rewards or penalties for tobacco or nicotine status. ${ }^{69}$ For some employers who implement tobacco/nicotine incentive programs, a standard policy is to offer a reward for those people who are tobacco and nicotine/cotinine free, or who are enrolled in, or have recently completed a qualified tobacco cessation program. Note that the regulations specifically acknowledge that tobacco cessation "sometimes requires a cycle of failure and renewed effort." 69 Therefore, incentives eligibility cannot be contingent on success, but rather relate only to the completion of a cessation program or some other reasonable alternative.
A positive nicotine test, whether due to tobacco use, nicotine replacement therapy such as gum or a patch, or the use of e-cigarettes, would prevent an individual from earning any incentive unless they met the reasonable alternative standard by completing a qualified cessation program, and/or have provided a physician statement, indicating that stopping the use of tobacco/nicotine or completing the cessation program was medically inadvisable or unreasonably difficult because of a medical issue or that the positive cotinine test result was due to the use of an NRT. The physician may join in a request for a different cessation method or indicate that a waiver of the requirement is appropriate.

Employers should be aware that the Equal Employment Opportunity Commission has filed several lawsuits against employers using health contingent incentive designs, indicating that they believe ACA wellness provisions do not satisfy the nondiscrimination tenets of the Americans with Disabilities Act. Further guidance from the agency should be forthcoming to help employers factor in other federal laws as they design financial incentives.

Finally, note that the ACA regulations for small groups (businesses with 50 employees or fewer) define tobacco use (for purposes of underwriting and rating variables) as "the use of tobacco on average of four or more times per week within no longer than the past six months." Furthermore, tobacco use must be defined in terms of when a tobacco product was last used. Tobacco includes all tobacco products; however, religious or ceremonial uses of tobacco (eg, by American Indians and Alaska Natives) are specifically exempt under the final rule. Note that the final wellness regulations did not adopt this definition, so employers in small groups should be aware that definitions used for application and underwriting reasons may differ from those used for insurance-based incentives.

\section{Additional Considerations for Employers}

\section{Regional Health Care Exchanges}

The new health insurance exchanges may play a greater role in population health management as some employers shift their employees to the exchanges for health care coverage. Health care plans sold through the exchanges should include comprehensive tobacco cessation services that also address e-cigarettes within their coverage. Further analysis will determine whether there are differences between private and public health exchanges in the content of their wellness offerings and how they are positioned for beneficiaries. Employers should advocate for the tobacco cessation benefits they want to see offered in the exchanges and 
should decide which exchanges provide the most effective programs in support of their employees' health and well-being.

\section{Collaboration With Labor Organizations}

Labor organizations are important, valuable, and culturally relevant partners in addressing e-cigarettes at the workplace. This is especially true considering that most unionized workers support their union in bargaining for restrictions on workplace smoking and for the availability of cessation programs. ${ }^{71,72}$ As such, joint labormanagement actions to address tobacco and e-cigarettes at the workplace are important as policy development, adoption, and implementation are all part of the bargaining process. ${ }^{73}$ There are many supportive actions labor organizations can take to help reduce worker exposure to nicotine and tobaccorelated products. Examples of specific actions include (1) providing members with the latest practical educational information about e-cigarettes; (2) advocating for tobacco bans at the workplace and including e-cigarettes as part of the definition of tobacco products; (3) advocating against the inclusion of e-cigarettes as a covered benefit for tobacco users because they are not an approved FDA cessation product; and (4) working collaboratively with management to address ecigarettes and broader tobacco policies at the workplace.

\section{Reaching Families and Raising Awareness}

Where employers extend their health promotion programs to the families of employees, they should raise awareness and include education around e-cigarettes and cessation therapy. It is especially important to reach children and adolescents where education may prevent initiation of a nicotine habit. Messages and programming should be tailored to the appropriate audience and families should be encouraged to use the tobacco cessation and prevention resources offered by the employer.

\section{Employers as Role Models}

Tobacco policy development may be influenced by a number of factors, including employer size, the type of employer, and the typical work environment. Ideally, policy development and tobacco cessation initiatives should be tailored to the specific needs of each employer's workforce, addressing both occupational safety and general health needs in a comprehensive way. Employers have a powerful opportunity to model effective tobacco policy and to offer best-practice, comprehensive cessation benefits and programming for employees and their family members that serve as a model in the community. Employers can also help the field gain a better understanding of best practices, thereby helping inform further research around effective policy for e-cigarettes and other emerging tobacco products.

\section{HOSPITAL SYSTEMS AS EMPLOYERS AND COMMUNITY INFLUENCERS}

Hospitals, by virtue of their mission, "lead the way and serve as role models for healthy living and fitness for their communities." 74 In a 2010 survey of hospitals conducted by the American Hospital Association, $76 \%$ of respondents indicated that they had a tobacco-free campus and $79 \%$ offered smoking cessation programs for their employees. ${ }^{74}$ Hospitals have increasingly seen the use of e-cigarettes by patients in their rooms and on the hospital grounds, and by visitors and staff. There are many resources available for hospital systems that want to adopt a $100 \%$ tobacco-free campus policy and incorporate a prohibition on the use of ecigarettes. ${ }^{75}$ Such policies can help with patient, employee, and community cessation efforts, lower maintenance and cleaning costs, and can increase job productivity. ${ }^{14}$ They also create a healthy environment for patients, visitors, and staff and allow hospitals to continue to serve as role models for their communities.

\section{EDUCATIONAL INSTITUTIONS AS EMPLOYERS AND INFLUENTIAL ENVIRONMENTS FOR YOUNG ADULTS}

The American College Health Association (ACHA) recommends that e-cigarettes be included in campus tobacco-free polices. ${ }^{76}$ Tobacco-free environments have led to substantial reductions in smoking prevalence, the number of people exposed to secondhand smoke, and the amount of tobacco products consumed. Such tobacco-free policies have transformed social norms around smoking in public and have made smoking a socially undesirable activity. College and university campuses are important environments for implementing robust policies and establishing social norms as a foundation for healthy behaviors in the young adult population. Colleges and universities as employers should be leaders in implementing model tobacco policies. The ACHA recommends that tobacco use be prohibited on all college and university grounds, college/university-owned or leased properties, and in campus-owned, leased, or rented vehicles. ${ }^{76}$ We concur with this recommendation.

\section{CONCLUSION}

E-cigarettes are a new, emerging, and increasingly popular product in the marketplace. They present challenges for employers as they establish their workplace tobacco policies, programming, and cessation services. This guidance is developed from expert consensus and may evolve as new research emerges and we gain a greater understanding of the long-term health impact of e-cigarettes and their effect on social norms and nicotine addiction. The organizations and researchers involved in writing this statement will continue to monitor the impact of these products on personal health, public health, employee health and safety, workplace culture, access to comprehensive cessation services, and the inclusion of e-cigarettes in laws, regulations, and organizational policy.

\section{REFERENCES}

1. Foulds J, Veldheer S, Berg A. Electronic cigarettes (e-cigs): views of aficionados and clinical/public health perspectives. Int J Clin Pract. 2011;65:1037-1042.

2. Zhu SH, Sun JY, Bonnevie E, et al. Four hundred and sixty brands of e-cigarettes and counting: implications for product regulation. Tob Control. 2014;23(suppl 3):iii3-iii9.

3. Grana R, Benowitz N, Glantz SA. Ecigarettes: a scientific review. Circulation. 2014;129:1972-1986.

4. Deeming Tobacco Products to Be Subject to the Federal Food, Drug, and Cosmetic Act, as Amended by the Family Smoking Prevention and Tobacco Control Act. Regulations on the Sale and Distribution of Tobacco Products and Required Warning Statements for Tobacco Products. 79 Fed Regist. 23141. April 25, 2014;21:CFR Parts 1100, 1140, 1143.

5. Biener L, Hargraves JL. A longitudinal study of electronic cigarette use in a populationbased sample of adult smokers: association with smoking cessation and motivation to quit. Nicotine Tob Res. 2014;17:127-133.

6. McRobbie H, Bullen C, Hartmann-Boyce J, Hajek P. Electronic cigarettes for smoking cessation and reduction. Cochrane Database Syst Rev. 2014;12:CD010216.

7. Grana RA, Popova L, Ling PM. A longitudinal analysis of electronic cigarette use and smoking cessation. JAMA Intern Med. 2014;174:812-813.

8. Gilbert HA. Inventor; Gilbert Herbert A, Assignee. US Patent 3,200,819. Smokeless nontobacco cigarette. August 17, 1965.

9. Rose SW, Barker DC, D'Angelo H, et al. The availability of electronic cigarettes in U.S. retail outlets, 2012: results of two national studies. Tob Control. 2014;23(suppl 3):iii10-6.

10. American Legacy Foundation. Vaporized: ECigarettes, Advertising, and Youth. Washington, DC: American Legacy Foundation; May 2014. Available at: http://legacyforhealth.org/ content/download/4542/63436/version/1/file/ LEG-Vaporized-E-cig_Report-May2014.pdf. Accessed January 5, 2015.

11. Rodgman A, Perfetti TA. The Chemical Components of Tobacco and Tobacco Smoke. 1st ed. Boca Raton, FL: CRC Press; 2009.

12. Ingrebrethsen B. Aerosol studies of cigarette smoke. Rec Adv Tob Science. 1986;12:54-142.

13. Office of the Surgeon General. The Health Consequences of Smoking-50 Years of 
Progress: A Report of the Surgeon General. Rockville, MD: US Department of Health and Human Services; 2014. Available at: http://www.surgeongeneral.gov/library/ reports/50-years-of-progress/full-report.pdf. Accessed January 5, 2015.

14. Committee on Secondhand Smoke Exposure and Acute Coronary Events; Institute of Medicine. Secondhand Smoke Exposure and Cardiovascular Effects: Making Sense of the Evidence. Washington, DC: The National Academies Press; 2009.

15. Hecht SS, Yuan JM, Hatsukami D. Applying tobacco carcinogen and toxicant biomarkers in product regulation and cancer prevention. Chem Res Toxicol. 2010;23:1001-1008.

16. Conklin DJ, Haberzettl P, Prough RA, Bhatnagar A. Glutathione-S-transferase P protects against endothelial dysfunction induced by exposure to tobacco smoke. Am J Physiol Heart Circ Physiol. 2009;296:H1586-H1597.

17. Conklin DJ, Barski OA, Lesgards JF, et al. Acrolein consumption induces systemic dyslipidemia and lipoprotein modification. Toxicol Appl Pharmacol. 2010;243:1-12.

18. Sithu SD, Srivastava S, Siddiqui MA, et al. Exposure to acrolein by inhalation causes platelet activation. Toxicol Appl Pharmacol. 2010;248:100-110.

19. Srivastava S, Sithu SD, Vladykovskaya E, et al. Oral exposure to acrolein exacerbates atherosclerosis in apoE-null mice. Atherosclerosis. 2011;215:301-308.

20. Haussmann HJ. Use of hazard indices for a theoretical evaluation of cigarette smoke composition. Chem Res Toxicol. 2012;25:794-810.

21. Hecht SS, Carmella SG, Kotandeniya D, et al. Evaluation of toxicant and carcinogen metabolites in the urine of e-cigarette users versus cigarette smokers [published online ahead of print October 21, 2014]. Nicotine Tob Res.

22. Werley MS, McDonald P, Lilly P, et al. Nonclinical safety and pharmacokinetic evaluations of propylene glycol aerosol in SpragueDawley rats and Beagle dogs. Toxicology. 2011;287:76-90.

23. US Food and Drug Administration. Select Committee on GRAS Substances (SCOGS) Opinion: Propylene Glycol. Available at: http://www.fda.gov/Food/Ingredients PackagingLabeling/GRAS/SCOGS/ ucm261045.htm. Published April 18, 2013. Accessed January 5, 2015.

24. Hutzler C, Paschke M, Kruschinski S, Henkler F, Hahn J, Luch A. Chemical hazards present in liquids and vapors of electronic cigarettes. Arch Toxicol. 2014;88:1295-1308.

25. US Centers for Disease Control and Prevention. The emergency response safety and health database: ethylene glycol. Available at: http://www.cdc.gov/niosh/ershdb/Emergency ResponseCard_29750031.html. Published May 12, 2011. Accessed January 5, 2015.

26. Etter JF, Zather E, Svensson S. Analysis of refill liquids for electronic cigarettes. Addiction. 2013;108:1671-1679.

27. Stepanov I, Jensen J, Hatsukami D, Hecht SS. New and traditional smokeless tobacco: comparison of toxicant and carcinogen levels. Nicotine Tob Res. 2008;10:1773-1782.

28. Uchiyama S, Inaba Y, Kunugita N. Determination of acrolein and other carbonyls in cigarette smoke using coupled silica cartridges impregnated with hydroquinone and 2,4-dinitrophenylhydrazine. J Chromatogr A. 2010;1217:4383-4388

29. Fuoco FC, Buonanno G, Stabile L, Vigo P. Influential parameters on particle concentration and size distribution in the mainstream of ecigarettes. Environ Pollut. 2014;184:523-529.

30. Ingebrethsen BJ, Cole SK, Alderman SL. Electronic cigarette aerosol particle size distribution measurements. Inhal Toxicol. 2012;24:976-984.

31. Brook RD, Rajagopalan S, Pope CA III, et al. American Heart Association Council on Epidemiology and prevention, council on the Kidney in Cardiovascular Disease, Council on Nutrition, Physical Activity and Metabolism. Particulate matter air pollution and cardiovascular disease: an update to the scientific statement from the American Heart Association. Circulation. 2010;121:2331-2378.

32. Vardavas CI, Anagnostopoulos N, Kougias M, Evangelopoulou V, Connolly GN, Behrakis PK. Short-term pulmonary effects of using an electronic cigarette: impact on respiratory flow resistance, impedance, and exhaled nitric oxide. Chest. 2012;141:1400-1406.

33. Flouris AD, Chorti MS, Poulianiti KP, et al. Acute impact of active and passive electronic cigarette smoking on serum cotinine and lung function. Inhal Toxicol. 2013;25:91-101.

34. Farsalinos K, Polosa R. Safety evaluation and risk assessment of electronic cigarettes as tobacco cigarette substitutes: a systematic review. Ther Adv Drug Safety. 2014;5:67-86.

35. Polosa R, Morjaria J, Caponnetto $P$, et al. Effect of smoking abstinence and reduction in asthmatic smokers switching to electronic cigarettes: evidence for harm reversal. Int J Environ Res Public Health. 2014;11: 4965-4977.

36. Monroy A, Smith S, Raji M. Paroxysmal atrial fibrillation following electronic cigarette use in an elderly woman. Clin Geriatr. 2012;20:28-32.

37. Barrington-Trimis JL, Samet JM, McConnell R. Flavorings in electronic cigarettes: an unrecognized respiratory health hazard? JAMA. 2014:312:2493-2494.

38. King BA, Alam S, Promoff G, Arrazola R, Dube SR. Awareness and ever-use of electronic cigarettes among U.S. adults, 2010 2011. Nicotine Tob Res. 2013;15:1623-1627.

39. Pepper JK, Brewer NT. Electronic nicotine delivery system (electronic cigarette) awareness, use, reactions and beliefs: a systematic review. Tob Control. 2014:23:375-384.

40. Regan AK, Promoff G, Dube SR, Arrazola R. Electronic nicotine delivery systems: adult use and awareness of the "e-cigarette" in the USA. Tob Control. 2013;22:19-23.

41. Richardson A, Williams V, Rath J, Villanti $\mathrm{AC}$, Vallone D. The next generation of users: prevalence and longitudinal patterns of tobacco use among US young adults. Am J Public Health. 2014;104:1429-1436.

42. Bunnell RE, Agaku IT, Arrazola RA, et al. Intentions to smoke cigarettes among neversmoking U.S. middle and high school electronic cigarette users, National Youth Tobacco Survey, 2011-2013. Nicotine Tob Res. 2015; 17:228-235.
43. World Health Organization Framework Convention on Tobacco Control. Electronic nicotine delivery systems. Available at: http://apps.who.int/gb/fctc/PDF/cop6/FCTC_ COP6_10-en.pdf. Published July 21, 2014. Accessed January 5, 2015.

44. Bhatnagar A, Whitsel LP, Ribisl KM, et al. American Heart Association Advocacy Coordinating Committee, Council on Caradiovascular and Stroke Nursing, Council on Clinical Cardiology, and Council on Quality of Care and Outcomes Research. Electronic cigarettes: a policy statement from the American Heart Association. Circulation. 2014;130:1418-1436.

45. American Association of Poison Control Centers. E-cigarette devices and liquid nicotine [Web site]. Available at: http://www.aapcc.org/ alerts/e-cigarettes./ Accessed January 6, 2015.

46. Mohney G. First child's death from liquid nicotine reported as "vaping" gains popularity. $A B C$ News. December 12, 2014. Available at: http://www.rtnz.com/first-childsdeath-from-liquid-nicotine-as-vaping-grows/. Accessed January 5, 2015.

47. Oberg M, Jaakkola MS, Woodward A, Peruga A, Pruss-Ustun A. Worldwide burden of disease from exposure to second-hand smoke: a retrospective analysis of data from 192 countries. Lancet. 2011;377:139-146.

48. Schober W, Szendrei K, Matzen W, et al. Use of electronic cigarettes (e-cigarettes) impairs indoor air quality and increases FeNO levels of e-cigarette consumers. Int $J$ Hyg Environ Health. 2014;217:628-637.

49. Czogala J, Goniewicz ML, Fidelus B, et al. Secondhand exposure to vapors from electronic cigarettes. Nicotine Tob Res. 2014; $16: 655-662$

50. Schripp T, Markewitz D, Uhde E, Salthammer $\mathrm{T}$. Does e-cigarette consumption cause passive vaping? Indoor Air. 2013;23:25-31.

51. Action on Smoking and Health. ASH briefing: electronic cigarettes. Available at: http://www .ash.org.uk/files/documents/ASH_715.pdf. Published November 2014. Accessed January $5,2015$.

52. European Commission. Revision of the tobacco products directive. Available at: http:// ec.europa.eu/health/tobacco/docs/dir_201440 _en.pdf. Published April 3, 2014. Accessed January 5, 2015

53. Gourdet CK, Chriqui JF, Chaloupka FJ. A baseline understanding of state laws governing e-cigarettes. Tob Control. 2014;23(suppl 3):iii37-iii40.

54. Adams S. Should e-cigarettes be allowed in the workplace. Forbes. April 29, 2014.

55. American Nonsmokers' Rights Foundation. US State and local laws regulating use of electronic cigarettes. Available at: http:// www.no-smoke.org/pdf/ecigslaws.pdf. Published 2014. Accessed January 5, 2015

56. World Health Organization. Backgrounder on WHO report on regulation of e-cigarettes and similar products [Web site]. Available at: http://www.who.int/nmh/events/2014/ backgrounder-e-cigarettes/en/. Published August 26, 2014. Accessed January 6, 2015.

57. Slavit W. What Employers Need to Know About Electronic Cigarettes. Washington, DC: National Business Group on Health; 2011. 
58. ChangeLab Solutions. Regulating toxic vapor: a policy guide to electronic smoking devices. Available at: http://changelabsolutions .org/sites/default/files/Regulating_Toxic_Vapor -FINAL-20140630.pdf. Published June 2014. Accessed January 6, 2015.

59. Fairchild AL, Bayer R, Colgrove J. The renormalization of smoking? E-cigarettes and the tobacco “endgame." N Engl J Med. 2014;370: 293-295.

60. McMillen RS, Wilson R, K. Use of electronic cigarettes among parents. Paper presented at: Pediatric Academic Societies Annual Meeting; May 4, 2014; Vancouver, British Columbia, Canada.

61. US Department of Health and Human Services. Preventive services covered under the Affordable Care Act [Web site]. Available at: http://www.hhs.gov/healthcare/ facts/factsheets/2010/07/preventive-serviceslist.html. Published 2012. Accessed January 6, 2015.

62. US Preventive Services Task Force. Counseling and interventions to prevent tobacco use and tobacco-caused disease in adults and pregnant women. Available at: http://www .uspreventiveservicestaskforce.org/uspstf/ uspstbac2.htm. Published 2010. Accessed January 6, 2015.

63. Community Preventive Services Task Force. Worksite health promotion [Web site]. Available at: http://www.thecommunityguide.org/ worksite/index.html. Published 2014. Accessed January 6, 2015.

64. US Department of Labor. FAQs about Affordable Care Act Implementation (Part XIX). Available at: http://www.dol.gov/ebsa/faqs/ faq-aca19.html. Published 2014. Accessed January 6, 2015.
65. Shaw A. E-cigarettes in the workplace: a policy toolkit to manage this new risk. Available at: http://www.xperthr.com/pages/ e-cigarette-policy-toolkit/. Published 2014. Accessed January 6, 2015.

66. Mayo Clinic. Test ID: NICOU nicotine and metabolites, urine. Available at: http://www .mayomedicallaboratories.com/test-catalog/ Clinical+and+Interpretive/82510. Accessed January 6, 2015.

67. South Carolina Code of Laws. Clean Indoor Air Act of 1990. Title 44, Chapter 95, Sections 10-60. Available at: http://www.scstatehouse. gov/code/t44c095.php. Accessed January 6, 2015.

68. National Business Group on Health. The trouble with tobacco testing. Interview with LuAnn Heinen conducted October 10, 2012. Available at: http://www.businessgroup health.org/pressroom/pressClipping.cfm?ID= 987. Accessed January 6, 2015.

69. Internal Revenue Service. Department of the Treasury; Employee Benefits Security Administration, Department of Labor. Incentives for Nondiscriminatory Wellness Programs in Group Health Plans: Final Rule. Fed Regist. 2013;78:FR 33157:33157-33192. Available at: http://www.federalregister.gov/ articles/2013/06/03/2013-12916/incentivesfor-nondiscriminatory-wellness-programsin-group-health-plans. Accessed January 6, 2015.

70. Consensus Statement of the Health Enhancement Research Organization, American College of Occupational and Environmental Medicine, American Cancer Society/American Cancer Society Cancer Action Network, American Diabetes Association, American Heart Association. Guidance for a reasonably designed, employer-sponsored wellness program using outcomes-based incentives. J Occup Environ Med. 2012;54:889896.

71. Mitchell RJ, Weisman SR, Jones RM, Erickson D. The role of labor organizations in tobacco control: what do unionized workers think? Am J Health Promot. 2009;23: 182-186.

72. WorkSHIFTS. Survey of Minnesota Union Members. St. Paul, MN: Tobacco Law Center; 2003.

73. Sorensen G, Youngstrom R, Maclachlan C, et al. Labor positions on worksite tobacco control policies: a review of arbitration cases. J Public Health Policy. 1997;18: 433-452.

74. American Hospital Association. 2010 Longrange policy committee, Bluford JW III, chair. A Call to Action: Creating a Culture of Health. Chicago, IL: American Hospital Association; 2011.

75. Americans for Nonsmokers' Rights. Hospitals and healthcare facilities [Web site] Available at: http://www.no-smoke.org/going smokefree.php?id=449. Accessed January 6 , 2015.

76. American College Health Association. Position Statement on Tobacco on College and University Campuses. Hanover, MD: American College Health Association; November 2011. Available at: http://www.acha.org/ Publications/docs/Position_Statement_on_ Tobacco_Nov2011.pdf. Accessed January 6, 2015.

77. Sottera vs. FDA. US Court of Appeals, District of Columbia. 627 F $3 \mathrm{~d} 891$. 2010. 